\title{
Correction to: Roles of E3 ubiquitin ligases in gastric cancer carcinogenesis and their effects on cisplatin resistance
}

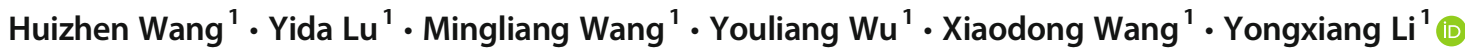 \\ Published online: 21 April 2021 \\ (C) Springer-Verlag GmbH Germany, part of Springer Nature 2021
}

Correction to: Journal of Molecular Medicine (2021) 99:193-212

https://doi.org/10.1007/s00109-020-02015-5

The correct assigned reference citations in Tables 2 and 3 is shown in this paper.

Moreover, we noticed that the reference 122 was incorrect in the online version, which is repetitive with the reference 112 , but the reference 122 was correct in the author proof version. Therefore, the reference 122 should be revised as follows:

122. Li LQ, Pan D, Chen H, Zhang L, Xie WJ (2016) F-box protein FBXL2 inhibits gastric cancer proliferation by ubiquitin-mediated degradation of forkhead box M1. FEBS Lett 590(4):445-452

Publisher's note Springer Nature remains neutral with regard to jurisdictional claims in published maps and institutional affiliations.

The online version of the original article can be found at https://oi.org/ 10.1007/s00109-020-02015-5

Yongxiang Li

liyongxiang@ahmu.edu.cn

1 Department of General Surgery, The First Affiliated Hospital of Anhui Medical University, Hefei 230022, China 
Table 2 Oncogenic E3 ligases in GC tumorigenesis

\begin{tabular}{|c|c|c|c|}
\hline Types & Sub-types & Genes & References \\
\hline \multirow[t]{25}{*}{ RINGs } & \multirow{18}{*}{ monomers } & UHRF1 & [38-42] \\
\hline & & RNF2 & [43] \\
\hline & & RNF6 & [44] \\
\hline & & RNF31 & [45] \\
\hline & & RNF38 & [46] \\
\hline & & RNF185 & [47] \\
\hline & & TRIM14 & {$[48]$} \\
\hline & & TRIM23 & [49] \\
\hline & & TRIM24 & {$[50-52]$} \\
\hline & & TRIM25 & [53] \\
\hline & & TRIM29 & {$[54-56]$} \\
\hline & & TRIM32 & {$[57,58]$} \\
\hline & & TRIM37 & [59] \\
\hline & & TRIM44 & [60] \\
\hline & & TRIM59 & [61] \\
\hline & & MKRN1 & [62] \\
\hline & & MARCH8 & [63] \\
\hline & & UBR5 & {$[64]$} \\
\hline & heterodimers & MDM2 & [65-68] \\
\hline & \multirow{6}{*}{$\begin{array}{l}\text { Cullin-RING ligases } \\
\text { (CRLs) }\end{array}$} & Cullin1 & [69] \\
\hline & & CACUL1 & {$[70,71]$} \\
\hline & & RBX1 & {$[72,73]$} \\
\hline & & SKP2 (FBXL1) & {$[74-76]$} \\
\hline & & FBXO2 & [77] \\
\hline & & FBXO6 & {$[78]$} \\
\hline \multirow[t]{2}{*}{ HECTs } & & WWP1 & [79-81] \\
\hline & & SMURF1 & {$[82]$} \\
\hline
\end{tabular}

Brown indicates that monomer E3 ligases exert oncogenic roles in GC tumorigenesis, and brick red indicates that CRLs act as oncoproteins for GC

Table 3 E3 ligases exert tumor suppressive and uncertain roles in GC tumorigenesis

\begin{tabular}{|c|c|c|c|c|}
\hline The roles in GC & Types & Sub-types & Genes & References \\
\hline \multirow[t]{16}{*}{ Tumor suppressor } & \multirow[t]{15}{*}{ RINGs } & \multirow[t]{5}{*}{ monomers } & RNF43 & {$[97-101]$} \\
\hline & & & RNF180 & [102-108] \\
\hline & & & RNF181 & [109] \\
\hline & & & ZNRF3 & {$[110]$} \\
\hline & & & TRIM15 & {$[111]$} \\
\hline & & homodimers & CHIP & [112-114] \\
\hline & & \multirow{6}{*}{$\begin{array}{l}\text { Cullin-RING ligases } \\
\text { (CRLs) }\end{array}$} & FBXW7 & [115-120] \\
\hline & & & $\beta-\operatorname{TrCP} 2$ (FBXW11) & [121] \\
\hline & & & FBXL2 & {$[122]$} \\
\hline & & & FBXL5 & {$[123,124]$} \\
\hline & & & FBXO8 & {$[125]$} \\
\hline & & & FBXO31 & {$[126,127]$} \\
\hline & & \multirow[t]{2}{*}{ Cul2-associated SOCS } & SOCS-1/SOCS-3 & {$[128]$} \\
\hline & & & SOCS-6 & {$[129,130]$} \\
\hline & & Cul3-associated BTB & SPOP & {$[131,132]$} \\
\hline & HECTs & & NEDD4-L & [133] \\
\hline \multirow[t]{5}{*}{ Uncertain roles } & \multirow[t]{4}{*}{ RINGs } & \multirow[t]{4}{*}{ monomers } & CHFR & [134-144] \\
\hline & & & Cbl-b & [145-149] \\
\hline & & & COP1 & {$[150,151]$} \\
\hline & & & TRIM31 & {$[152,153]$} \\
\hline & HECTs & & NEDD4-1 & [154-156] \\
\hline
\end{tabular}

Brown and brick red indicate that monomer E3 ligases and cullin-ring ligases (CRLs) exert tumor suppressive roles in GC tumorigenesis, and light green indicates monomer E3 ligases act uncertain roles in GC pathogenesis 